\title{
Characterization of charge-carrier dynamics in thin oxide layers on silicon by second harmonic generation
}

\author{
Yu. D. Glinka, W. Wang, S. K. Singh, Z. Marka, S. N. Rashkeev, Y. Shirokaya, R. Albridge, \\ S. T. Pantelides, and N. H. Tolk \\ Department of Physics and Astronomy, Vanderbilt University, Nashville, Tennessee 37235 \\ G. Lucovsky \\ Department of Physics, North Carolina State University, Raleigh, North Carolina 27695
}

(Received 11 February 2002; published 23 April 2002)

\begin{abstract}
First measurements of time-dependent second-harmonic generation $(\mathrm{SHG})$ at a $\mathrm{Si} /(\mathrm{ZrO})_{x}\left(\mathrm{SiO}_{2}\right)_{1-x}$ interface show a behavior that is drastically different from similar measurements at $\mathrm{Si} / \mathrm{SiO}_{2}$ interfaces. We suggest that in $\mathrm{Si} / \mathrm{SiO}_{2}$ only electron injection is important, while both electrons and holes contribute to the dynamics at the $\mathrm{Si} /\left(\mathrm{ZrO}_{2}\right)_{x}\left(\mathrm{SiO}_{2}\right)_{1-x}$ interface. Multiphoton excitation occurs in $\mathrm{Si}$ for all oxides, and involves direct interband transitions. The marked difference between the two systems is related to the population of multiphoton excited states in $\mathrm{Si}$, the corresponding conduction- and valence-band offsets, and trapping/detrapping processes in the oxides. Our measurements confirm the existence of an initial built-in field at the interface.
\end{abstract}

DOI: 10.1103/PhysRevB.65.193103

PACS number(s): 73.50.Gr, 42.65.Ky, 73.40.-c, 77.55.+f

Recent advances in ultrafast laser technology and nonlinear optics have opened up new venues for fundamental studies of carrier injection dynamics at interfaces. Among these approaches, second harmonic generation (SHG) analysis has several advantages. It is contactless, nonintrusive, and can be used for in situ measurements. SHG analysis is a sensitive tool for systems with broken inversion symmetry such as surfaces and interfaces. The fact that a nonoscillatory electric field in a material can greatly enhance SHG signals at an interface was employed in an electric-field-induced secondharmonic (EFISH) analysis which recently attracted particular attention. ${ }^{1}$ The interfacial static electric field arising from charge separation depends strongly on the dynamics of the charge carriers of the materials, i.e., EFISH measurements provide unique information on electronic structure, local fields, symmetry, and carrier dynamics at interfaces. ${ }^{2,3}$ The relationship between the SHG signals and a slowly varying electric field at the interface can be expressed as

$$
I(2 \omega) \propto \mid \chi^{(2)}+\chi^{(3)}\left(E_{0}-\left.E(t)\right|^{2} I^{2}(\omega),\right.
$$

where $I(\omega)$ is the intensity of the incident laser light, $E_{0}$ is the initial dipole electric field, and $E(t)$ is the slowly varying time-dependent field, both at the interface. $\chi^{(2)}$ and $\chi^{(3)}$ are the interfacial second- and third-order susceptibilities.

In this Brief Report, we present time-dependent SHG measurements that exhibit strong contributions from both the electron and hole injection processes. This is achieved at an interface between $\mathrm{Si}$ and a high- $k$ dielectric $\left(\mathrm{ZrO}_{2}\right)_{x}\left(\mathrm{SiO}_{2}\right)_{1-x}$ oxide layer. Earlier SHG studies on photoexcitation at the $\mathrm{Si} / \mathrm{SiO}_{2}$ interface concluded that electrons play a crucial and exclusive role in the development of the interfacial electric fields. ${ }^{4}$ Our measurements elucidate the important role of holes in the dynamical processes, leading to charge separation at the interface. ${ }^{5}$

High- $k$ dielectrics were investigated in recent years as a possible replacement for silicon dioxide at $\mathrm{Si} / \mathrm{SiO}_{2}$ interfaces. ${ }^{6-8}$ High- $k$ dielectric materials were found to sig- nificantly diminish the leakage current caused by electron tunneling. $\left(\mathrm{ZrO}_{2}\right)_{x}\left(\mathrm{SiO}_{2}\right)_{1-x}$ is distinguished from other high- $k$ materials, because it does not react with silicon and creates thermally and chemically stable interfaces. ${ }^{8}$

In our measurements we used amorphous $\left(\mathrm{ZrO}_{2}\right)_{x}\left(\mathrm{SiO}_{2}\right)_{1-x}$ films grown on $\mathrm{Si}(100)$ substrates at North Carolina State University using remote-controlled plasma-enhanced chemical vapor deposition. The oxide thickness is estimated to be of the order of several hundred $\AA{ }^{9}{ }^{9}$ The band gap was measured to be approximately $5.6 \mathrm{eV}$. We compared the results of SHG measurements in these samples with measurements performed in samples from Lucent Technologies [40 $\AA$ of thermally grown oxide film on $\mathrm{Si}(100)]$. The SHG experiment used a standard configuration. 150-fs pulses (700 $\mathrm{nm}<\lambda<900 \mathrm{~nm}$ ) from a modelocked Ti:sapphire laser (Coherent Mira 900) was focused on the sample. The interval between pulses was $13 \mathrm{~ns}$, the power in the pulse was about $50 \mathrm{GW} / \mathrm{cm}^{2}$, and the beam spot on the sample was of the order of $10 \mu \mathrm{m}$ in diameter. The SHG signal was optically separated from the reflected fundamental beam, and measured by a photon-multiplier tube through the photon counter. All the measurements have been carried out in air at room temperature.

Time-dependent SHG curves for the $\mathrm{Si} /\left(\mathrm{ZrO}_{2}\right)_{x}\left(\mathrm{SiO}_{2}\right)_{1-x}$ and $\mathrm{Si} / \mathrm{SiO}_{2}$ systems, for a photon energy $1.56 \mathrm{eV}$ and for different laser powers, are compared in Fig. 1. For laser powers below $450 \mathrm{~mW}$, the time-dependent SHG signals are basically the same for both materials. For higher powers, the curves arising from the $\mathrm{Si} / \mathrm{SiO}_{2}$ interface continue to increase gradually with time (toward saturation), while the curves from the $\mathrm{Si} /\left(\mathrm{ZrO}_{2}\right)_{x}\left(\mathrm{SiO}_{2}\right)_{1-x}$ system rise rapidly, reach a maximum, and gradually decrease. In the most general case, these curves can be described by an expression with one or two intensity-dependent time constants. The SHG signal at $t=0\left(\Delta_{1}\right)$ grows nearly quadratically vs laser power, thus confirming the validity of Eq. (1).

In addition to the primary dynamics effects shown in Fig. 1 , our measurements show that at lower powers (less than 


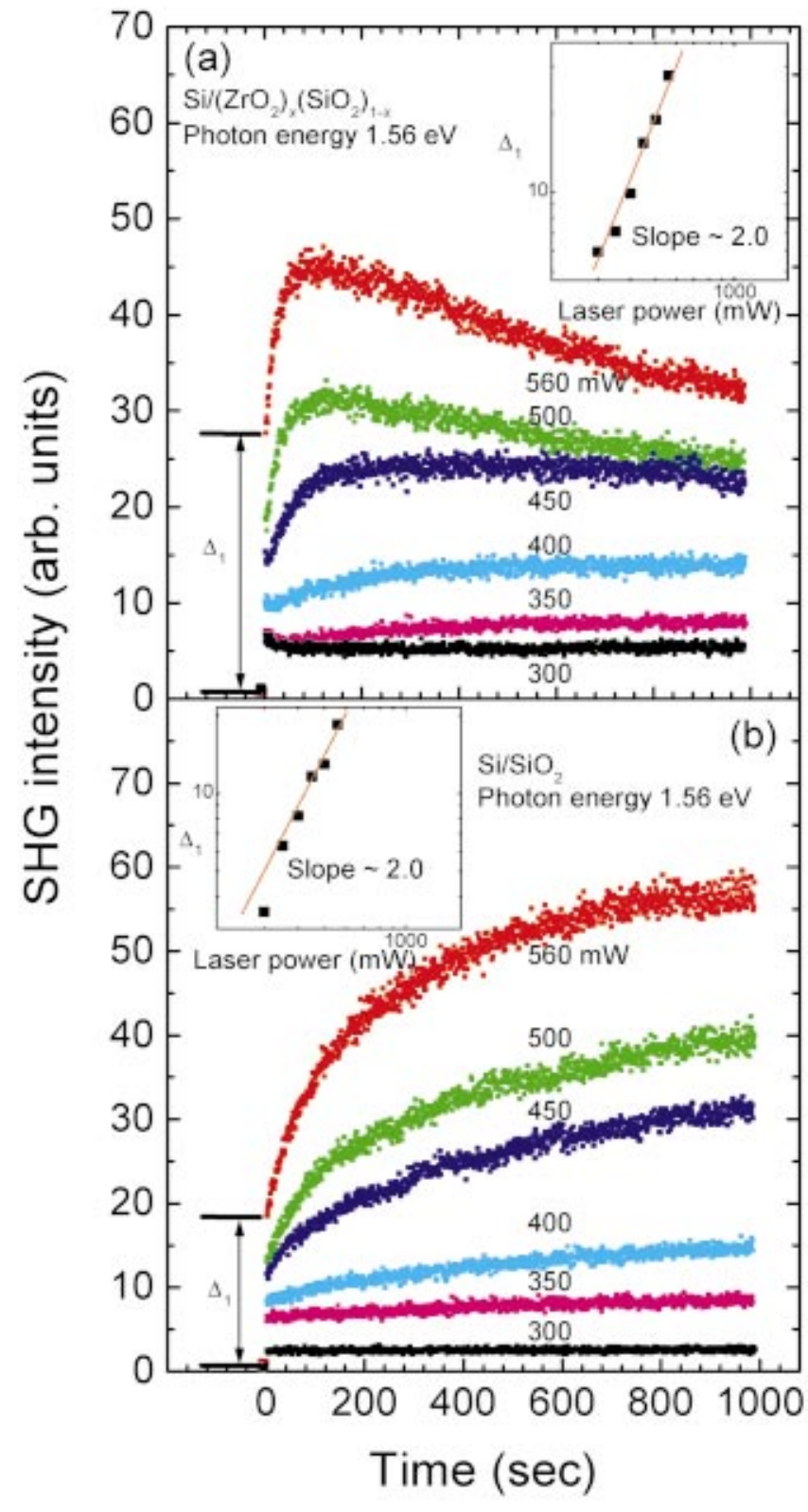

FIG. 1. (Color) SHG signals from the interface between Si and $\left(\mathrm{ZrO}_{2}\right)_{x}\left(\mathrm{SiO}_{2}\right)_{1-x}(\mathrm{a})$, and $\mathrm{SiO}_{2}$ (b). The photon energy is $1.56 \mathrm{eV}$, the laser power varies between 300 and $560 \mathrm{~mW}$. The insets show the dependence of the SHG signal at $t=0\left(\Delta_{1}\right)$ vs laser power on a $\log -\log$ scale.

$400 \mathrm{~mW}$ ) there is an initial decrease in the SHG signal for the zirconia system immediately after the laser radiation is applied. Figure 2 shows this behavior of the SHG signal for different photon energies and for different laser powers. We suggest that this decrease in the signal is related to the fact that there is an initial dipole field at the interface. This is supported by previous nonoptical measurements at the $\mathrm{Si} /\left(\mathrm{ZrO}_{2}\right)_{x}\left(\mathrm{SiO}_{2}\right)_{1-x}$ interface. ${ }^{13}$ In this picture, the charge accumulated near the interface is negative in $\mathrm{Si}$ and positive in the oxide, i.e., the initial interfacial electric field is directed toward the Si substrate. As electrons are injected, an electric field due to charge separation begins to develop but in the opposite direction. The net field then initially de- (a)

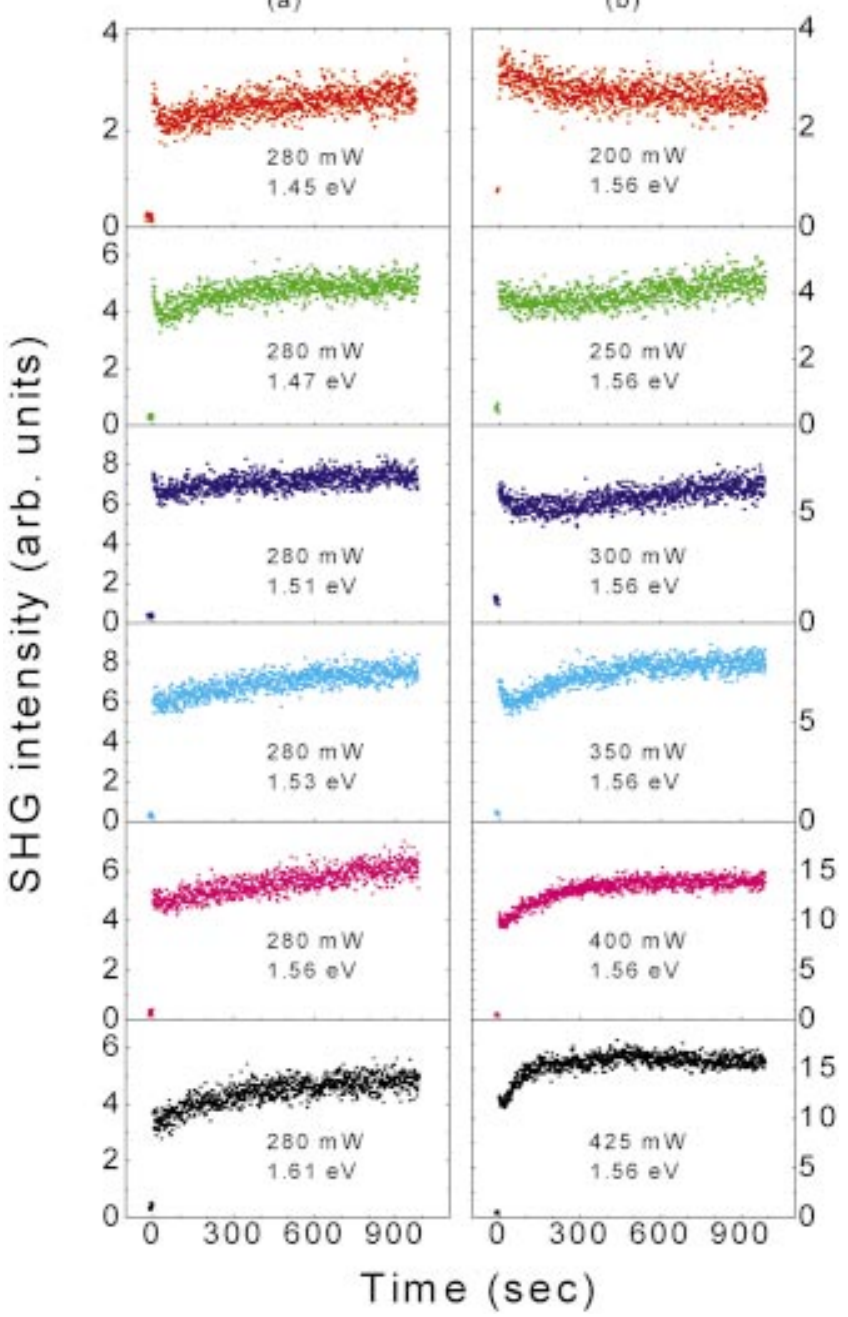

FIG. 2. (Color) Time-dependent SHG signal at the $\mathrm{Si} /\left(\mathrm{ZrO}_{2}\right)_{x}\left(\mathrm{SiO}_{2}\right)_{1-x}$ interface: (a) For different photon energies and for fixed laser power $(280 \mathrm{~mW})$ and (b) for photon energy 1.56 $\mathrm{eV}$ and for different power of the laser.

creases to some minimum, and then begins to increase. In other words, one can say that the initial decrease and subsequent increase of the SHG signal occur due to an interplay between the initial dipole static dipole field and the timedependent field at the interface caused by the injection and trapping of charge carriers in the oxide. The cross-term in Eq. (1) describes this behavior.

The time-dependent field at the interface also depends on the photon energy [Fig. 2(a)]. The initial fast decrease of the SHG signal practically disappears when the photon energy grows from 1.45 to $1.61 \mathrm{eV}$. When the photon energy increases, the kinetic energy of electrons injected into the oxide is higher, and we expect them to be trapped faster. As a result, the time to reverse the initial electric field at the interface becomes shorter, and the SHG minimum is not easily observed.

Figure 3 shows the dependence of zero-electric-field SHG signal vs the photon energy in the $\mathrm{Si} /\left(\mathrm{ZrO}_{2}\right)_{x}\left(\mathrm{SiO}_{2}\right)_{1-x}$ system. Since the increase of the SHG intensity with a twophoton energy corresponds to the edge of the direct interband 


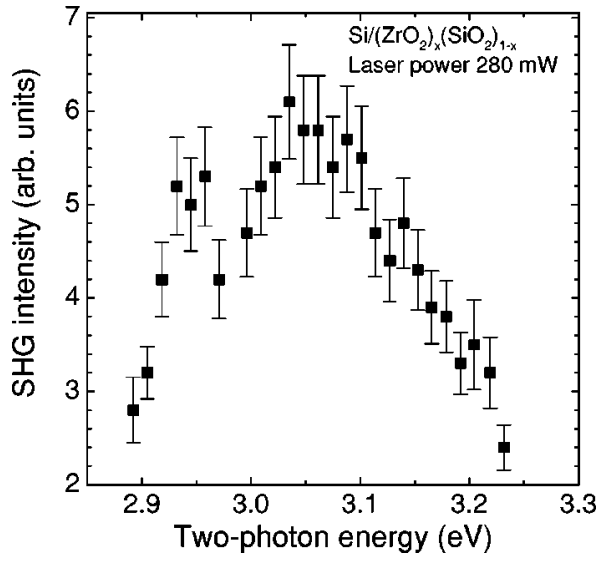

FIG. 3. Zero-electric-field SHG signal at the $\mathrm{Si} /\left(\mathrm{ZrO}_{2}\right)_{x}\left(\mathrm{SiO}_{2}\right)_{1-x}$ interface vs the two-photon energy of the laser light.

transitions in $\mathrm{Si}$, we confirm that the SHG process occurs through resonant interband transitions.

A straightforward interpretation of the time-dependent SHG measurements from $\mathrm{Si} / \mathrm{SiO}_{2}$ takes into account the energies of band offsets for the valence and conduction bands. The barrier for electrons between the silicon valence band and the $\mathrm{SiO}_{2}$ conduction band at a $\mathrm{Si} / \mathrm{SiO}_{2}$ interface is about $4.3 \mathrm{eV}$ and, therefore, for a photon energy $1.56 \mathrm{eV}$, the injection of electrons from $\mathrm{Si}$ into silicon dioxide is assumed to be a third-order process. ${ }^{4}$ The injected electrons traveling across the oxide, are eventually trapped at the oxygenambient surface of the oxide, thus creating a slowly varying electric field across the interface. The corresponding barrier for holes is $5.8 \mathrm{eV}$, and thus one needs at least four photons with the same energy for injection. Since third-order processes are much more probable than fourth-order processes, it is clear that electron injection dominates.

The electronic structure at the $\mathrm{Si} /\left(\mathrm{ZrO}_{2}\right)_{x}\left(\mathrm{SiO}_{2}\right)_{1-x}$ interface is significantly different from the $\mathrm{Si} / \mathrm{SiO}_{2}$ interface (Fig. 4). Calculations give only 1.5 and $3.4 \mathrm{eV}$ for the conductionand valence-band offsets of the stoichiometric $\mathrm{ZrSiO}_{4}$ compound (zircon), and 3.3 and $1.5 \mathrm{eV}$ for $\mathrm{ZrO}_{2}{ }^{10}$ This indicates that the band gap is mostly defined by the transition-metal $d$ levels. X-ray photoemission measurements (XPS) do not

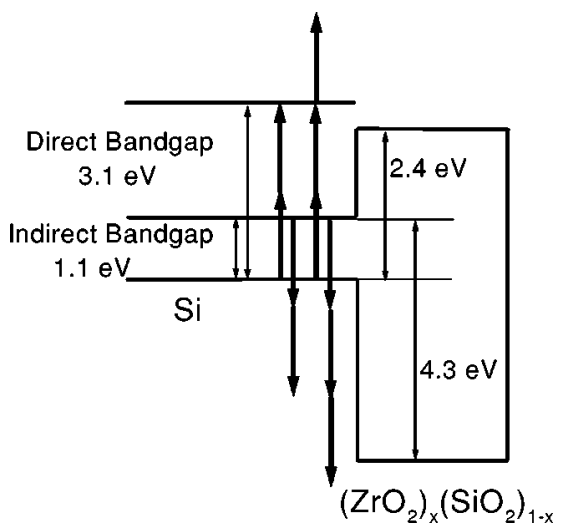

FIG. 4. Band diagram and carrier dynamics at the $\mathrm{Si} /\left(\mathrm{ZrO}_{2}\right)_{x}\left(\mathrm{SiO}_{2}\right)_{1-x}$ interface. show any evidence of $\mathrm{Zr}-\mathrm{Si}$ bond formation. ${ }^{8}$ $\left(\mathrm{ZrO}_{2}\right)_{x}\left(\mathrm{SiO}_{2}\right)_{1-x}$ can be considered as parallel chains of $\mathrm{ZrO}_{2}$ and $\mathrm{SiO}_{2}$ structural unit molecules mixed in different proportions. $^{11}$ A recent XPS measurement for the $\mathrm{Si} /\left(\mathrm{ZrO}_{2}\right)_{x}\left(\mathrm{SiO}_{2}\right)_{1-x}$ interface gave $1.3 \mathrm{eV}$ for the conduction-band offset. ${ }^{12}$

In the most general case, charge injection in the oxide consists of two processes, namely, (i) multiphoton excitation of electrons and holes in $\mathrm{Si}$ (the oxide is transparent for incident laser light), and thus the excitation process does not depend on the nature of the oxide; and (ii) the transport of electrons and holes across the interface, assuming that the initial energy of the excited carrier is higher than the corresponding barrier. From energy considerations, we can assume that a two-photon process will inject electrons into the $\left(\mathrm{ZrO}_{2}\right)_{x}\left(\mathrm{SiO}_{2}\right)_{1-x}$ oxide, but one needs at least three photons to inject holes.

However, such an interpretation is certainly not sufficient, and one must take into account the intensity of the light as well. The multiphoton carrier excitations in $\mathrm{Si}$ are independent of the nature of the oxide, and depend only on the intensity of the laser beam. For a given intensity, an excitation of a particular order should dominate. This follows from the expression for the probability of an $n$-photon process,

$$
W^{(n)}=\sigma^{(n)} I^{n}
$$

where $I$ is the intensity of the beam, and $\sigma^{(n)}$ is the cross section. The power $50 \mathrm{GW} / \mathrm{cm}^{2}$ of the laser during the pulse corresponds to $I \sim 10^{29} \mathrm{~cm}^{-2} \mathrm{sec}^{-1}$, and one can conclude that for realistic values of the cross sections the nonlinear processes are, at least, not weaker than the linear process. ${ }^{14,15}$ To determine the order of the process that dominates, one should obtain detailed information about the cross sections for excitation processes of different orders in Si. Excitations of both the electrons and holes in Si by multiple-photon processes occur only during the 150 -fs pulse. This time is shorter than other energy-relaxation times in the system.

The injection and trapping of carriers into the oxide occur primarily in the time interval between pulses (13 ns). These carriers after injection can contribute to charge separation across the interface. In this case, since the electrons and holes are injected at different rates, this dynamic process changes the local charge distribution and consequently also the slowly varying electric field at the interface. In order to move away from the interface, carriers should have an energy that is higher than the barrier related to the offset plus the hopping activation barrier. The hopping activation barrier for holes $(1.5 \mathrm{eV})$ is much higher than that for electrons $(0.1$ $\mathrm{eV}){ }^{16}$ This is consistent with measurements of trapping cross sections in amorphous $\mathrm{SiO}_{2}$ (the trapping cross sections for holes were measured to be 5-6 orders of magnitude larger than for electrons). ${ }^{17,18}$ Therefore, the energy value of an offset is clearly not sufficient to determine the threshold energy for carrier injection and trapping.

Most electron-hole pairs created during the pulse will relax and recombine between the pulses. In pure silicon, Auger recombination processes probably dominate over direct radiative recombination (see Ref. 19, and references therein). 
Both experiment and calculations show that in both $n$ - and $p$-doped Si the lifetime for the Auger recombination is in the range $10^{-5}-10^{-10} \mathrm{~s}$ when the carrier concentration is in the range $10^{18}-10^{20} \mathrm{~cm}^{-3}$. Other mechanisms may also contribute, but it is apparent that only carriers that are trapped in the oxide in the time interval between pulses will contribute to the slowly varying electric field at the interface.

The similarity of the time-dependent SHG curves for the two oxides at laser powers below $400 \mathrm{~mW}$ indicates that in both cases we deal with the injection of electrons only (Fig. 1). In the $\mathrm{Si} / \mathrm{SiO}_{2}$ system, the electron-hole pairs in $\mathrm{Si}$ are excited by a third-order process. In this case electrons can go into the oxide through the barrier, but holes cannot. In the $\mathrm{Si} /\left(\mathrm{ZrO}_{2}\right)_{x}\left(\mathrm{SiO}_{2}\right)_{1-x}$ system, electrons can be injected even in a second-order process, but one needs a third-order process to inject holes. When the laser power is relatively low, the injection of holes does not play a significant role, and thus we have similar curves for both systems. We suggest that the absence of a hole injection process at the $\mathrm{Si} /\left(\mathrm{ZrO}_{2}\right)_{x}\left(\mathrm{SiO}_{2}\right)_{1-x}$ interface at low laser power indicates that we are below an intensity-dependent threshold for third- order injection. Thus at some critical power the third-order process begins to contribute significantly, and hole injection begins to provide a substantial contribution. At this point the SHG signal begins to gradually decrease due to a reduction in a net interfacial field.

In summary, we have shown that both the electron and hole injection processes contribute to the observed timedependent SHG signal from the $\mathrm{Si} /\left(\mathrm{ZrO}_{2}\right)_{x}\left(\mathrm{SiO}_{2}\right)_{1-x}$ system. In addition, our measurements confirm the existence of an initial dipole electric field at the interface. We suggest that the intensity-dependent multiphoton excitation processes in $\mathrm{Si}$ that initiate the charge separation at the interface are independent of the oxide, and are related to direct interband transitions. The SHG technique has been shown to be extremely sensitive to injection/trapping processes at interfaces, and, consequently, holds great promise for a nondestructive characterization of electron-hole dynamics at semiconductor/oxide interfaces.

This work was supported by the Office of Naval Research under Grants No. N00014-94-1-1023, N00014-94-1-0995, and N00014-96-1-1286.
${ }^{1}$ G. Lüpke, Surf. Sci. Rep. 35, 75 (1999).

${ }^{2}$ J. F. McGilp, J. Phys. D 29, 1812 (1996).

${ }^{3}$ O. A. Aktsipetrov et al., Phys. Rev. B 60, 8924 (1999).

${ }^{4}$ J. Bloch, J. G. Mihaychuk, and H. M. van Driel, Phys. Rev. Lett. 77, 920 (1996).

${ }^{5}$ Our earlier publication [W. Wang et al., Phys. Rev. Lett. 81, 4224 (1998)] on coupled electron-hole dynamics was incorrect with regard to hole injection, due to a misinterpretation of some temperature dependent effects in the SHG measurements.

${ }^{6}$ S. A. Campbell et al., IEEE Trans. Electron Devices 44, 104 (1997).

${ }^{7}$ I. C. Kizilyalli, R. Y. S. Huang, and P. K. Roy, IEEE Electron Device Lett. 19, 423 (1998).

${ }^{8}$ G. D. Wilk, R. M. Wilk, R. M. Wallace, and J. M. Anthony, J. Appl. Phys. 87, 484 (2000).

${ }^{9}$ D. M. Wolfe et al., in Ultrathin $\mathrm{SiO}_{2}$ and High-K Materials for ULSI Gate Dielectrics, edited by H. R. Huff, M. L. Green, T. Hattori, G. Lucovsky, and C. A. Richter, MRS Symposia Proceedings No. 567 (Materials Research Society, Pittsburgh, 1999), p. 343.

${ }^{10}$ J. Robertson, J. Vac. Sci. Technol. B 18, 1785 (2000).

${ }^{11}$ L. Bragg, G. F. Claringbull, and W. H. Taylor, Crystal Structures of Minerals (Cornell University Press, Ithaca, NY, 1965), p. 185.

${ }^{12}$ S. Miyazaki, J. Vac. Sci. Technol. B 19, 2212 (2001).

${ }^{13}$ G. Lucovsky, J. C. Phillips, and M. F. Thorpe, in Proceedings of the Characterization and Metrology for USLI Technology, edited by D. G. Seiler, A. C. Diebold, T. J. Shaffner, R. McDonald, W. M. Bullis, P. J. Smith, and E. M. Secula, AIP Conf. Proc. No. 550 (AIP, New York, 2001), p. 154.

${ }^{14}$ Yu. D. Glinka, K.-W. Lin, H.-C. Chang, and S. H. Lin, J. Phys. Chem. B 103, 4251 (1999).

${ }^{15}$ L. J. Rothberg, D. P. Gerrit, and V. J. Vaida, J. Phys. Chem. 75, 4403 (1981).

${ }^{16}$ Y. Takemura, J. Ushio, T. Mariuzumi, K. Kubota, and M. Miyao, Mater. Sci. Semicond. Proc. 2, 253 (1999).

${ }^{17}$ D. J. DiMaria, in The Physics of $\mathrm{SiO}_{2}$ and Its Interfaces, edited by S. T. Pantelides (Pergamon, Elmsford, NY, 1978), pp. 160178.

${ }^{18}$ R. C. Hughes, E. P. EerNisse, and H. J. Stein, IEEE Trans. Nucl. Sci. NS-22, 2227 (1975).

${ }^{19}$ D. B. Laks, G. F. Neumark, A. Hangleiter, and S. T. Pantelides, Phys. Rev. Lett. 61, 1229 (1988); D. B. Laks, G. F. Neumark, and S. T. Pantelides, Phys. Rev. B 42, 5176 (1990). 